\title{
Semi-direct sums of Lie algebras and discrete integrable couplings
}

\author{
Wen-Xiu Ma* \\ Department of Mathematics, University of South Florida, Tampa, FL 33620-5700, USA \\ $\mathrm{Xi}-\mathrm{Xiang} \mathrm{Xu}^{\dagger}$ \\ College of Science, Shandong University of Science and Technology, Qingdao 266510, P.R. China \\ Yufeng Zhang $\ddagger$ \\ School of Mathematics, Liaoning Normal University, Dalian 116029, P.R. China
}

\begin{abstract}
A relation between semi-direct sums of Lie algebras and integrable couplings of lattice equations is established, and a practicable way to construct integrable couplings is further proposed. An application of the resulting general theory to the generalized Toda spectral problem yields two classes of integrable couplings for the generalized Toda hierarchy of lattice equations. The construction of integrable couplings using semi-direct sums of Lie algebras provides a good source of information on complete classification of integrable lattice equations.

Key words: Semi-direct sums of Lie algebras, Discrete spectral problems, Discrete zero curvature equations, Integrable couplings

PACS codes: 02.10.De, 02.30.Ik
\end{abstract}

\section{Introduction}

Integrable couplings have been receiving growing attention recently. A few ways to construct integrable couplings are presented by using perturbations [1, 2, 3], enlarging spectral problems [4, 5], and creating new loop Lie algebras [6, 17.

The problem of integrable couplings can be expressed as follows [2]: For a given integrable system, how can we construct a non-trivial system of differential equations which is still integrable and includes the original integrable system as a sub-system? Obviously, a

\footnotetext{
*Email: mawx@math.usf.edu (W.X. Ma), Tel: (813)974-9563, Fax: (813)974-2700

${ }^{\dagger}$ Email: xu_xixiang@sohu.com (X.X. Xu)

†Email: zhang_yfshandong@163.com (Y. Zhang)
} 
change of orders of equations in a system does not lose integrability of the system. Therefore, up to a permutation, an integrable coupling of a given integrable system $u_{t}=K(u)$ is given by a bigger and triangular system:

$$
u_{t}=K(u), v_{t}=S(u, v)
$$

The vector-valued function $S$ should satisfy the non-triviality condition $\partial S / \partial[u] \neq 0$, where $[u]=\left(u, D_{x} u, D_{x}^{2} u, \cdots\right)$ and $D_{x}^{n} u$ denotes a vector consisting of all derivatives of $u$ of order $n$ with respect to the space variable $x$. The above non-triviality condition means that the second sub-system involves the dependent variables of the first sub-system (i.e. the original system), and thus it guarantees that trivial diagonal systems with $S(u, v)=S(v)$ are not within our discussion.

A basic integrable coupling of an integrable system $u_{t}=K(u)$ is given by

$$
u_{t}=K(u), v_{t}=K^{\prime}(u)[v]
$$

which can be generated by a perturbation around a solution of the system $u_{t}=K(u)[1$. In the above system and elsewhere throughout this paper, $P^{\prime}(u)[v]$ denotes the Gateaux derivative of $P(u) \equiv P\left(u, D_{x} u, \cdots\right)$ with respect to $u$ in a direction $v$, i.e.,

$$
P^{\prime}(u)[v]=\left.\frac{\partial}{\partial \varepsilon} P(u+\varepsilon v)\right|_{\varepsilon=0}=\left.\frac{\partial}{\partial \varepsilon} P\left(u+\varepsilon v, D_{x} u+\varepsilon D_{x} v, \cdots\right)\right|_{\varepsilon=0} .
$$

Obviously, the second sub-system $v_{t}=K^{\prime}(u)[v]$ in the above integrable coupling (1.1) is linear with respect to $v$. Moreover, a symmetry $S(u)$ of the system $u_{t}=K(u)$ leads to a solution $(u, S(u))$ to the integrable coupling (1.1). However, the second component $v$ of a solution $(u, v)$ to the integrable coupling (1.1) is generally not a symmetry of the system $u_{t}=K(u)$. This is because $v$ satisfies the linearized system $v_{t}=K^{\prime}(u)[v]$ only for one solution, not for all solutions of the system $u_{t}=K(u)$. Therefore, the simple integrable coupling (1.1) is already a generalization of the symmetry problem. Another basic integrable coupling of an integrable system $u_{t}=K(u)$ reads as

$$
u_{t}=K(u), v_{t}=K^{\prime}(u)[v]+K(u)
$$

This system has a set of hereditary recursion operators [2]

$$
\Phi\left(\beta_{1}, \beta_{2}\right)=\left(\begin{array}{cc}
\beta_{1} \Phi(u) & 0 \\
\beta_{1} \Phi^{\prime}(u)[v]+\beta_{2} \Phi(u) & \beta_{1} \Phi(u)
\end{array}\right)
$$

with two arbitrary constants $\beta_{1}$ and $\beta_{2}$, if the original system $u_{t}=K(u)$ has a hereditary recursion operator $\Phi(u)$. Therefore, integrable couplings possess richer integrable structures than the original integrable systems. 
The study of integrable couplings provides clues towards complete classification of integrable systems. Let us first observe classification of square matrix spectral problems through the Jordan blocks under similar transformations of matrices. Each triangular Jordan block corresponds to an undecomposable sub-system in a given integrable system. Now, note that an arbitrary Lie algebra has a semi-direct sum structure of a solvable Lie algebra and a semi-simple Lie algebra [8], and we will see that semi-direct sums of Lie algebras can result in integrable couplings. These imply that the study of integrable couplings through semi-direct sums of Lie algebras is an inevitable step towards complete classification of integrable systems with an arbitrary number of components, from a point of view of Lie algebras.

The study of integrable couplings also generates interesting mathematical structures such as Lax pairs with several spectral parameters 9, 10, 2], integrable constrained flows with higher multiplicity [11, 12, local bi-Hamiltonian structures in higher dimensions [13] and hereditary recursion operators of higher order [2, 14]. Very recently, we have proposed a relation between semi-direct sums of Lie algebras and integrable couplings of continuous soliton equations, which provides an interesting and systematic approach to integrable couplings of continuous soliton equations [15]. In this paper, we would like to discuss the problem of discrete integrable couplings and develop a theory for constructing discrete integrable couplings by use of semi-direct sums of Lie algebras.

Throughout our discussion, we denote by $E$ the shift operator, write

$$
\left(E^{m} x\right)(n)=x^{(m)}(n)=x(m+n), \text { where } x: \mathbb{Z} \rightarrow \mathbb{R}, m, n \in \mathbb{Z},
$$

and adopt an inverse of the difference operator $E-1$ as follows

$$
(E-1)^{-1}=\frac{1}{2}\left(\sum_{k=-\infty}^{-1}-\sum_{k=0}^{\infty}\right) E^{k} .
$$

Let $G$ be a matrix Lie algebra with the standard Lie bracket $[A, B]=A B-B A$, and closed under matrix multiplication: $A B \in G$ for all $A, B \in G$. We assume that an integrable lattice equation (or system) of evolution type

$$
u_{t}=K(u)=K\left(u, E u, E^{-1} u, \cdots\right)
$$

is associated with $G$, where $u=u(n, t)$ is a dependent variable. More precisely, there is a pair of square matrices $U$ and $V$ in $G$, called a Lax pair, so that the discrete spatial matrix spectral problem

$$
E \phi=U \phi=U(u, \lambda) \phi
$$

and the associated discrete temporal matrix spectral problem

$$
\phi_{t}=V \phi=V\left(u, E u, E^{-1} u, \cdots ; \lambda\right) \phi,
$$


where $\lambda$ is a spectral parameter and $\phi$ is an eigenfunction, generate [16, 17] the integrable lattice equation (1.5) through their isospectral (i.e., $\lambda_{t}=0$ ) compatibility condition

$$
U_{t}=(E V) U-U V
$$

which is called a discrete zero curvature equation. In other words, we have

$$
U^{\prime}(u)[K]=(E V) U-U V,
$$

where $U^{\prime}(u)[K]$ denotes the Gateaux derivative as above. In a non-isospectral case, e.g., $\lambda_{t}=f(\lambda)$, then we have

$$
U^{\prime}(u)[K]+f U_{\lambda}=(E V) U-U V
$$

where $U_{\lambda}$ is a partial derivative of $U$ with respect to $\lambda$. Based on (1.6) and (1.7), the lattice equation (1.5) can often be solved by the inverse scattering transform (for example, see [18). There are also a few interesting Lie algebraic structures hidden behind the equation (1.10) (see 17] for more information). An integrable hierarchy and its master symmetry hierarchy usually correspond to the isospectral case and the non-isospectral case $\lambda_{t}=\lambda^{n}, n \geq 0$, respectively. These two hierarchies constitute a semi-direct sum of Lie algebras, each of which consists of symmetries in one hierarchy. The spatial matrix spectral problem (1.6) is our starting point in constructing discrete integrable couplings. The closure property of the Lie algebra $G$ under matrix multiplication guarantees that $(E V) U-U V$ is still in $G$ so that the discrete zero curvature equation (1.8) makes sense.

In what follows, we are going to establish a relation between semi-direct sums of Lie algebras and integrable couplings of lattice equations and a technically-practicable way to generate integrable couplings through semi-direct sums of Lie algebras. The resulting general theory will be used to generate two classes of integrable couplings for the generalized Toda hierarchy presented in 19. It will also be indicated that the construction of integrable couplings using semi-direct sums of Lie algebras provides a good source of information about classification of integrable lattice equations. A few concluding remarks will be given in the last section.

\section{Constructing integrable couplings using semi-direct sums of Lie algebras}

\subsection{Generating scheme}

Assume that the lattice equation (1.5) has a Lax pair $(U, V)$ in a matrix Lie algebra $G$ closed under matrix multiplication. 
To construct an integrable coupling of the lattice equation (1.5), we use semi-direct sums of Lie algebras to enlarge the original Lie algebra $G$. Take another matrix Lie algebra $G_{c}$ closed under matrix multiplication and then form a semi-direct sum $\bar{G}$ of $G$ and $G_{c}$ :

$$
\bar{G}=G \notin G_{c} .
$$

The notion of semi-direct sums means that $G$ and $G_{c}$ satisfy

$$
\left[G, G_{c}\right] \subseteq G_{c}
$$

where $\left[G, G_{c}\right]=\left\{[A, B] \mid A \in G, B \in G_{c}\right\}$. Obviously, $G_{c}$ is an ideal Lie sub-algebra of $\bar{G}$. The subscript $c$ here indicates a contribution to the construction of couplings. We also require that the closure property between $G$ and $G_{c}$ under matrix multiplication:

$$
G G_{c}, G_{c} G \subseteq G_{c}
$$

where $G_{1} G_{2}=\left\{A B \mid A \in G_{1}, B \in G_{2}\right\}$, to guarantee that a Lax pair from the semi-direct sum $\bar{G}$ can generate a coupling system. Note that the two different binary operations were used in the above closure properties in (2.2) and (2.3).

Now choose a pair of new Lax matrices in the semi-direct sum $\bar{G}$ of Lie algebras:

$$
\bar{U}=U+U_{c}, \bar{V}=V+V_{c}, U_{c}, V_{c} \in G_{c}
$$

and make a pair of enlarged discrete spatial matrix spectral problems

$$
\left\{\begin{array}{l}
E \bar{\phi}=\bar{U} \bar{\phi}=\bar{U}(\bar{u}, \lambda) \bar{\phi} \\
\bar{\phi}_{t}=\bar{V} \bar{\phi}=\bar{V}\left(\bar{u}, E \bar{u}, E^{-1} \bar{u} \cdots ; \lambda\right) \bar{\phi}
\end{array}\right.
$$

where the matrix $U_{c}$ in $\bar{U}$ introduces additional dependent variables and $\bar{u}$ consists of both the original dependent variables and the additional dependent variables. In addition, the matrix $U_{c}$ could depend on the spectral parameter $\lambda$, and the matrix $V_{c}$ in $\bar{V}$ really does almost in all cases. Based on the closure properties of $G, G_{c}$ and between $G$ and $G_{c}$, it is easy to see that

$$
\begin{aligned}
& (E \bar{V}) \bar{U}-\bar{U} \bar{V}=[(E V) U-U V] \\
& +\left\{\left[(E V) U_{c}-U_{c} V\right]+\left[\left(E V_{c}\right) U-U V_{c}\right]+\left[\left(E V_{c}\right) U_{c}-U_{c} V_{c}\right]\right\} \in G \notin G_{c} .
\end{aligned}
$$

Therefore, under $u_{t}=K(u)$, the corresponding enlarged discrete zero curvature equation

$$
\bar{U}_{t}=(E \bar{V}) \bar{U}-\bar{U} \bar{V}
$$

precisely presents

$$
\left\{\begin{array}{l}
U_{t}=(E V) U-U V \\
U_{c, t}=\left[(E V) U_{c}-U_{c} V\right]+\left[\left(E V_{c}\right) U-U V_{c}\right]+\left[\left(E V_{c}\right) U_{c}-U_{c} V_{c}\right] .
\end{array}\right.
$$


The first equation above is equivalent to the lattice equation (1.5), and hence, this is a coupling system for the lattice equation (1.5).

The whole construction process above shows that semi-direct sums of a given Lie algebra $G$ with new Lie algebras provide a great choice of candidates of integrable couplings for the lattice equation (1.5) generated from the Lie algebra $G$.

\section{$2.2 \quad$ Realizations by particular semi-direct sums}

To shed light on the above general scheme of constructing coupling systems, let us introduce the following particular class of semi-direct sums of Lie algebras:

$$
\begin{aligned}
& \bar{G}=G \notin G_{c}, G=\{\operatorname{diag}(\underbrace{A, \cdots, A}_{\mu}, \underbrace{0, \cdots, 0}_{\nu-\mu+1})\}, \\
& G_{c}=\left\{\left(\begin{array}{cccccc}
0 & & & & & \\
& \ddots & & & B_{i j} & \\
& & 0 & & & \\
& & & B_{\mu+1, \mu+1} & & \\
& 0 & & \ddots & \\
& & & & & B_{\nu+1, \nu+1}
\end{array}\right)\right\},
\end{aligned}
$$

where $A, B_{i i}$ are arbitrary square matrices, $A$ is of the same order as $U$ and the partitions of matrices in $G$ and $G_{c}$ are the same. Obviously, $B_{i j}, j \leq \mu$, are square but $B_{i j}, j \geq \mu+1$, may not; and all closure conditions of $G, G_{c}$ and between $G$ and $G_{c}$ under matrix multiplication are satisfied.

Define

$$
U_{G}:=\operatorname{diag}(\underbrace{U, \cdots, U}_{\mu}, \underbrace{0, \cdots, 0}_{\nu-\mu+1}), V_{G}:=\operatorname{diag}(\underbrace{V, \cdots, V}_{\mu}, \underbrace{0, \cdots, 0}_{\nu-\mu+1}) .
$$

Note that $U_{G}$ and $V_{G}$ in $G$ generate the same lattice equation as $U$ and $V$, and thus for integrable couplings, the corresponding enlarged spectral matrices $\bar{U}$ and $\bar{V}$ in the semi- 
direct sum $G \notin G_{c}$ can be chosen as

$$
\begin{aligned}
& \bar{U}=U_{G}+U_{G, c}:=\left(\begin{array}{cccccc}
U & & & & & \\
& \ddots & & & & \\
& & & & & \\
& & U & & & \\
& & & U_{\mu+1, \mu+1} & & \\
& & & & \ddots & \\
& 0 & & & & U_{\nu+1, \nu+1}
\end{array}\right) \\
& \bar{V}=V_{G}+V_{G, c}:=\left(\begin{array}{cccccc}
V & & & & & \\
& \ddots & & & & \\
& & & & \\
& & V & & & \\
& & & V_{\mu+1, \mu+1} & & \\
& & & & \ddots & \\
& 0 & & & & V_{\nu+1, \nu+1}
\end{array}\right),
\end{aligned}
$$

where the first two matrices $U_{G}$ and $V_{G}$ play the $(U, V)$-part and the second two matrices $U_{G, c}$ and $V_{G, c}$ play the $\left(U_{c}, V_{c}\right)$-part in the pair of $\bar{U}$ and $\bar{V}$ defined in (2.4). It is not difficult to see that the resulting coupling system (2.7) becomes

$$
\left\{\begin{array}{l}
U_{t}=(E V) U-U V, \\
\left(U_{i+1, i+1}\right)_{t}=\left(E V_{i+1, i+1}\right) U_{i+1, i+1}-U_{i+1, i+1} V_{i+1, i+1}, \quad \mu \leq i \leq \nu, \\
U_{i j, t}=\sum_{k=i}^{j}\left[\left(E V_{i k}\right) U_{k j}-U_{i k} V_{k j}\right], 1 \leq i<j \leq \nu+1,
\end{array}\right.
$$

where $U_{i i}=U$ and $V_{i i}=V, 1 \leq i \leq \mu$.

In particular, first, if we take

$$
\bar{U}=\left(\begin{array}{cccc}
U & U_{12} & \cdots & U_{1, \mu+1} \\
& \ddots & \ddots & \vdots \\
& & U & U_{\mu, \mu+1} \\
0 & & & 0
\end{array}\right), \bar{V}=\left(\begin{array}{cccc}
V & V_{12} & \cdots & V_{1, \mu+1} \\
& \ddots & \ddots & \vdots \\
& & V & V_{\mu, \mu+1} \\
0 & & & 0
\end{array}\right),
$$

then the coupling system (2.9) becomes

$$
\left\{\begin{array}{l}
U_{t}=(E V) U-U V, \\
U_{i j, t}=\sum_{k=i}^{j}\left[\left(E V_{i k}\right) U_{k j}-U_{i k} V_{k j}\right], 1 \leq i<j \leq \mu+1,
\end{array}\right.
$$


where $U_{i i}=U, V_{i i}=V, 1 \leq i \leq \mu$, and $U_{i i}=V_{i i}=0, i=\mu+1$. Second, if we take

$$
\bar{U}=\left(\begin{array}{cccc}
U & U_{a_{1}} & \cdots & U_{a_{\nu}} \\
& U & \ddots & \vdots \\
& & \ddots & U_{a_{1}} \\
0 & & & U
\end{array}\right), \bar{V}=\left(\begin{array}{cccc}
V & V_{a_{1}} & \cdots & V_{a_{\nu}} \\
& V & \ddots & \vdots \\
& & \ddots & V_{a_{1}} \\
0 & & & V
\end{array}\right),
$$

then the coupling system (2.9) becomes

$$
\left\{\begin{array}{l}
U_{t}=(E V) U-U V, \\
U_{a_{i}, t}=\sum_{k+l=i, k, l \geq 0}\left[\left(E V_{a_{k}}\right) U_{a_{l}}-U_{a_{l}} V_{a_{k}}\right], 1 \leq i \leq \nu,
\end{array}\right.
$$

where $U_{a_{0}}=U$ and $V_{a_{0}}=V$.

We remark that here we have just presented one class of semi-direct sums of Lie algebras, together with two specific examples. It is interesting to construct other possible realizations, especially those which could carry essential information for keeping integrable properties of the original lattice equations.

\subsection{Linearly dependent case on the spectral parameter}

Let us now assume that the spatial spectral matrix $U$ depends linearly on the spectral parameter $\lambda$ (see, for example, [17, 19, 20, 21]):

$$
U=U(u, \lambda)=\lambda U_{0}+U_{1}, \frac{\partial U_{0}}{\partial \lambda}=\frac{\partial U_{1}}{\partial \lambda}=0 .
$$

Consider two specific examples of the enlarged spatial spectral matrices introduced in the last sub-section:

$$
\bar{U}_{1}=\left(\begin{array}{cc}
U & U_{a} \\
0 & 0
\end{array}\right), \bar{U}_{2}=\left(\begin{array}{cc}
U & U_{a} \\
0 & U
\end{array}\right), \frac{\partial U_{a}}{\partial \lambda}=0 .
$$

Note that the sub-matrices $U_{a}$ in the above two enlarged spatial spectral matrices could be of different sizes. As in the continuous cases [4, 15], suppose that

$$
\bar{W}_{1}=\left(\begin{array}{cc}
W & W_{a} \\
0 & 0
\end{array}\right), \bar{W}_{2}=\left(\begin{array}{cc}
W & W_{a} \\
0 & W
\end{array}\right)
$$

with

$$
W=\sum_{i \geq 0} W_{i} \lambda^{-i}, W_{a}=\sum_{i \geq-n_{0}} W_{a, i} \lambda^{-i}, \frac{\partial W_{i}}{\partial \lambda}=0, \frac{\partial W_{a, i}}{\partial \lambda}=0,
$$

where $n_{0} \geq 0$ is a proper integer, solve the corresponding enlarged discrete stationary zero curvature equations

$$
\left(E \bar{W}_{i}\right) \bar{U}_{i}-\bar{U}_{i} \bar{W}_{i}=0, i=1,2,
$$


respectively.

Then for each $m \geq 0$, choose

$$
\bar{V}_{1}^{[m]}=\left(\begin{array}{cc}
V^{[m]} & V_{a}^{[m]} \\
0 & 0
\end{array}\right)=\left(\lambda^{m} \bar{W}_{1}\right)_{+}+\bar{\Delta}_{m}, \bar{\Delta}_{m}=\left(\begin{array}{cc}
\Delta_{m} & \Delta_{m, a} \\
0 & 0
\end{array}\right)
$$

where $\Delta_{m}$ and $\Delta_{m, a}$ do not depend on $\lambda$ and satisfy

$$
\left(E \Delta_{m}\right) U_{0}-U_{0} \Delta_{m}=0, U_{0} \Delta_{m, a}=0
$$

and choose

$$
\bar{V}_{2}^{[m]}=\left(\begin{array}{cc}
V^{[m]} & V_{a}^{[m]} \\
0 & V^{[m]}
\end{array}\right)=\left(\lambda^{m} \bar{W}_{2}\right)_{+}+\bar{\Delta}_{m}, \bar{\Delta}_{m}=\left(\begin{array}{cc}
\Delta_{m} & \Delta_{m, a} \\
0 & \Delta_{m}
\end{array}\right),
$$

where $\Delta_{m}$ and $\Delta_{m, a}$ do not depend on $\lambda$ and satisfy

$$
\left(E \Delta_{m}\right) U_{0}-U_{0} \Delta_{m}=0,\left(E \Delta_{m, a}\right) U_{0}-U_{0} \Delta_{m, a}=0
$$

The subscript + above denotes to select the polynomial part in $\lambda$. Based on (2.10) and (2.11) and using (2.15), we can directly show that the enlarged discrete zero curvature equations

$$
\bar{U}_{i, t_{m}}=\left(E \bar{V}_{i}^{[m]}\right) \bar{U}_{i}-\bar{U}_{i} \bar{V}_{i}^{[m]}, i=1,2,
$$

namely,

$$
\left\{\begin{array} { c } 
{ U _ { t _ { m } } = ( E V ^ { [ m ] } ) U - U V ^ { [ m ] } , } \\
{ U _ { a , t _ { m } } = ( E V ^ { [ m ] } ) U _ { a } - U V _ { a } ^ { [ m ] } , }
\end{array} \quad \text { and } \left\{\begin{array}{c}
U_{t_{m}}=\left(E V^{[m]}\right) U-U V^{[m]}, \\
U_{a, t_{m}}=\left(E V^{[m]}\right) U_{a}+\left(E V_{a}^{[m]}\right) U \\
-U V_{a}^{[m]}-U_{a} V^{[m]},
\end{array}\right.\right.
$$

present

$$
\left\{\begin{array}{l}
U_{t_{m}}=\left(\Delta_{m}\right)_{x}+\left[U_{0}, W_{m+1}\right]-\left[U_{1}, \Delta_{m}\right] \\
U_{a, t_{m}}=U_{0} W_{a, m+1}+\left(E \Delta_{m}\right) U_{a}-U_{1} \Delta_{m, a}
\end{array}\right.
$$

and

$$
\left\{\begin{aligned}
U_{t_{m}}= & \left(\Delta_{m}\right)_{x}+\left[U_{0}, W_{m+1}\right]-\left[U_{1}, \Delta_{m}\right] \\
U_{a, t_{m}}= & U_{0} W_{a, m+1}-\left(E W_{a, m+1}\right) U_{0} \\
& +\left(E \Delta_{m}\right) U_{a}-U_{a} \Delta_{m}+\left(E \Delta_{m, a}\right) U_{1}-U_{1} \Delta_{m, a}
\end{aligned}\right.
$$

respectively.

We remark that these two enlarged hierarchies in (2.18) and (2.19) share the enlarged discrete spectral problems

$$
E \bar{\phi}=\bar{U}_{1} \bar{\phi}, E \bar{\phi}=\bar{U}_{2} \bar{\phi}
$$


respectively. Thus, all lattice equations in each of the two enlarged hierarchies can possess infinitely many common conserved densities except the original ones (see [23, 24, 25] for a few concrete examples). Moreover, one can construct a specific non-degenerate bilinear form on $\bar{G}$ with the invariance property, to present Hamiltonian structures of the enlarged lattice equations by a generalized trace identity. The detailed analysis on those integrable properties will be left to a future publication.

To sum up, each system of lattice equations in the hierarchy (2.18) or (2.19) can provide an integrable coupling for its first sub-system of lattice equations. In the next section, we will only discuss two examples of constructing enlarged lattice hierarchies, in the generalized Toda case presented in [19].

\section{Integrable couplings of the generalized Toda hier- archy}

\subsection{The generalized Toda equations}

Let us here recall the generalized Toda hierarchy [19]. The corresponding discrete spatial spectral problem reads

$$
E \phi=U(u, \lambda) \phi, U(u, \lambda)=\left(\begin{array}{cc}
0 & 1 \\
(\alpha \lambda+\beta) r & \lambda+s
\end{array}\right), u=\left(\begin{array}{l}
r \\
s
\end{array}\right)
$$

where $\lambda$ is a spectral parameter, and $\alpha$ and $\beta$ are two arbitrary constants satisfying $\alpha^{2}+\beta^{2} \neq$ 0 . When $\alpha=0$ and $\beta=-1$, (3.1) becomes the Toda spectral problem [16].

Its stationary discrete zero curvature equation

$$
(E W) U-U W=0
$$

has the solution

$$
W=\left(\begin{array}{cc}
a & b \\
(\alpha \lambda+\beta) c & -a
\end{array}\right)
$$

with

$$
a=\sum_{i \geq 0} a_{i} \lambda^{-i}, b=\sum_{i \geq 0} b_{i} \lambda^{-i}, c=\sum_{i \geq 0} c_{i} \lambda^{-i},
$$

where the coefficients are defined by the initial conditions:

$$
a_{0}=-\frac{1}{2}, b_{0}=0, c_{0}=0,
$$


and the recursion relation:

$$
\left\{\begin{array}{l}
c_{i+1}-r b_{i+1}^{(1)}=0, \\
b_{i+1}^{(1)}+s b_{i}^{(1)}+\left(a_{i}^{(1)}+a_{i}\right)=0, \\
\left(a_{i+1}^{(1)}-a_{i+1}\right)+s\left(a_{i}^{(1)}-a_{i}\right) \\
+\alpha\left(r b_{i+1}-c_{i+1}^{(1)}\right)+\beta\left(r b_{i}-c_{i}^{(1)}\right)=0,
\end{array} \quad i \geq 0,\right.
$$

which are all difference polynomials in $u$ with respect to the lattice variable $n$. Under the initial-value conditions

$$
\left.a_{1}\right|_{u=0}=\left.c_{1}\right|_{u=0}=0,\left.a_{i}\right|_{u=0}=\left.b_{i}\right|_{u=0}=\left.c_{i}\right|_{u=0}=0, i \geq 2,
$$

the recursion relation (3.4) uniquely determine the lattice functions $a_{i}, b_{i}$ and $c_{i}, i \geq 1$. The first few lattice functions are

$$
\left\{\begin{array}{l}
a_{1}=\alpha r, b_{1}=1, c_{1}=r \\
a_{2}=-\alpha^{2} r^{(1)} r-\alpha^{2} r^{2}-\alpha^{2} r r^{(-1)}-\alpha r s-\alpha r s^{(-1)}+\beta r \\
b_{2}=-\alpha r-\alpha r^{(-1)}-s^{(-1)}, c_{2}=-r s-\alpha r^{2}-\alpha r r^{(1)} .
\end{array}\right.
$$

As usual, choose that

$$
V_{m}=\left(\begin{array}{cc}
\left(\lambda^{m} a\right)_{+} & \left(\lambda^{m} b\right)_{+} \\
(\alpha \lambda+\beta)\left(\lambda^{m} c\right)_{+} & -\left(\lambda^{m} a\right)_{+}
\end{array}\right), m \geq 0 .
$$

Then it follows from (3.4) that

$$
\left(E V_{m}\right) U-U V_{m}=\left(\begin{array}{cc}
0 & -b_{m+1}^{(1)} \\
(\alpha \lambda+\beta) c_{m+1} & \beta\left(c_{m}^{(1)}-r b_{m}\right)-s\left(a_{m}^{(1)}-a_{m}\right)
\end{array}\right) .
$$

Take a modification

$$
\Delta_{m}=\left(\begin{array}{cc}
b_{m+1} & 0 \\
0 & 0
\end{array}\right)
$$

and define the temporal spectral matrices

$$
V^{[m]}=V_{m}+\Delta_{m}, m \geq 0
$$

Then, a direct calculation leads to the following matrix:

$$
\left(E V^{[m]}\right) U-U V^{[m]}=\left(\begin{array}{cc}
0 & 0 \\
(\alpha \lambda+\beta)\left(c_{m+1}-r b_{m+1}\right) & \beta\left(c_{m}^{(1)}-r b_{m}\right)-s\left(a_{m}^{(1)}-a_{m}\right)
\end{array}\right) .
$$

This is consistent with $U_{t_{m}}$, and thus, making the evolution laws

$$
\phi_{t_{m}}=V^{[m]} \phi, m \geq 0,
$$


the compatibility conditions

$$
U_{t_{m}}=\left(E V^{[m]}\right) U-U V^{[m]}, m \geq 0
$$

of the discrete spatial spectral problem (3.1) and the associated discrete temporal spectral problems (3.7) give rise to the following hierarchy of lattice equations

$$
\left\{\begin{array}{l}
r_{t_{m}}=c_{m+1}-r b_{m+1}, \\
s_{t_{m}}=-\alpha\left(c_{m+1}^{(1)}-r b_{m+1}\right)+\left(a_{m+1}^{(1)}-a_{m+1}\right),
\end{array} \quad m \geq 0 .\right.
$$

This generalized Toda hierarchy is Liouville integrable [19], and its Hamiltonian structure leads to infinitely many conservation laws and symmetries for every system in the hierarchy.

Obviously, the first nonlinear lattice equation in the hierarchy is

$$
\left\{\begin{array}{l}
r_{t_{1}}=r\left(s^{(-1)}-s\right)+\alpha r\left(r^{(-1)}-r^{(1)}\right) \\
s_{t_{1}}=\alpha s\left(r-r^{(1)}\right)+\beta\left(r^{(1)}-r\right)
\end{array}\right.
$$

When $\alpha=0$ and $\beta=-1$, (3.9) becomes the Toda lattice equation [22]:

$$
r_{t_{1}}=r\left(s^{(-1)}-s\right), s_{t_{1}}=r-r^{(1)}
$$

and when $\alpha=1$ and $\beta=0$, (3.9]) becomes the following lattice equation presented in [26]:

$$
r_{t_{1}}=r\left(s^{(-1)}-s\right)+r\left(r^{(-1)}-r^{(1)}\right), s_{t_{1}}=s\left(r-r^{(1)}\right) \text {. }
$$

The lattice equation (3.11) is linearly independent of the Toda lattice equation (3.10). There exist a voluminous literature on the Toda lattice equation, and its generalizations and solution structures (for example, see [27]-32]).

\subsection{Integrable couplings from specific semi-direct sums}

The generalized Toda spectral problem (3.1) linearly depends on the spectral parameter $\lambda$, and thus we can write

$$
U=\left(\begin{array}{cc}
0 & 1 \\
(\alpha \lambda+\beta) r & \lambda+s
\end{array}\right)=U_{0} \lambda+U_{1}, U_{0}=\left(\begin{array}{cc}
0 & 0 \\
\alpha r & 1
\end{array}\right), U_{1}=\left(\begin{array}{cc}
0 & 1 \\
\beta r & s
\end{array}\right) .
$$

We will also see that there is a difference between the two cases of $\alpha=0$ and $\alpha \neq 0$ in computing integrable couplings.

Let us first consider the semi-direct sum of Lie algebras of $3 \times 3$ matrices:

$G \in G_{c}, G=\left\{\left(\begin{array}{ll}A & 0 \\ 0 & 0\end{array}\right) \mid A \in \mathbb{C}\left[\lambda, \lambda^{-1}\right] \otimes M_{2 \times 2}\right\}, G_{c}=\left\{\left(\begin{array}{ll}0 & B \\ 0 & 0\end{array}\right) \mid B \in \mathbb{C}\left[\lambda, \lambda^{-1}\right] \otimes M_{2 \times 1}\right\}$, 
where $\mathbb{C}\left[\lambda, \lambda^{-1}\right] \otimes M_{m \times n}=\operatorname{span}\left\{\lambda^{k} A \mid k \in \mathbb{Z}, A \in M_{m \times n}\right\}$. In this case, $G_{c}$ is an Abelian ideal of $G \notin G_{c}$. We define the corresponding enlarged spatial spectral matrix as

$$
\bar{U}=\bar{U}(\bar{u}, \lambda)=\left(\begin{array}{cc}
U & U_{a} \\
0 & 0
\end{array}\right) \in G \notin G_{c}, U_{a}=U_{a}(v)=\left(\begin{array}{l}
v_{1} \\
v_{2}
\end{array}\right),
$$

where $v_{1}$ and $v_{2}$ are new dependent variables and

$$
v=\left(v_{1}, v_{2}\right)^{T}, \bar{u}=\left(u^{T}, v^{T}\right)^{T}=\left(r, s, v_{1}, v_{2}\right)^{T} .
$$

Upon setting

$$
\bar{W}=\left(\begin{array}{cc}
W & W_{a} \\
0 & 0
\end{array}\right), W_{a}=W_{a}(\bar{u}, \lambda)=\left(\begin{array}{l}
e \\
f
\end{array}\right),
$$

where $W$ is a solution to $(E W) U-U W=0$, defined by (3.3), the corresponding enlarged discrete stationary zero curvature equation $(E \bar{W}) \bar{U}-\bar{U} \bar{W}=0$ becomes

$$
(E W) U_{a}-U W_{a}=0
$$

which is equivalent to

$$
W_{a}=W U^{-1} U_{a}
$$

namely,

$$
\left\{\begin{array}{l}
f=a^{(1)} v_{1}+b^{(1)} v_{2}, \\
(\alpha \lambda+\beta) r e=(\alpha \lambda+\beta) c^{(1)} v_{1}-a^{(1)} v_{2}-(\lambda+s) f .
\end{array}\right.
$$

This system determines a solution for $e$ and $f$ as follows

$$
e=\sum_{i \geq-n_{0}} e_{i} \lambda^{-i}, f=\sum_{i \geq 0} f_{i} \lambda^{i}
$$

where $n_{0}=1$ if $\alpha=0$ and $n_{0}=0$ if $\alpha \neq 0$ (see (2.14) for introduction of $n_{0}$ ). Now define the enlarged temporal spectral matrix as

$$
\bar{V}^{[m]}=\left(\begin{array}{cc}
V^{[m]} & V_{a}^{[m]} \\
0 & 0
\end{array}\right), V_{a}^{[m]}=\left(\lambda^{m} W_{a}\right)_{+}+\Delta_{m, a}, m \geq 0,
$$

where $V^{[m]}$ is defined as in (3.6) . To satisfy (2.16), choose $\Delta_{m, a}$ as

$$
\Delta_{m, a}=\left(\begin{array}{c}
h_{m} \\
-\alpha r h_{m}
\end{array}\right), h_{m}-\text { arbitrary, } m \geq 0 .
$$

Then based on (3.15), we can compute that

$$
\begin{aligned}
& \left(E V^{[m]}\right) U_{a}-U V_{a}^{[m]}=\left(E V_{m}\right) U_{a}-U\left(\lambda^{m} W_{a}\right)_{+}+\left(E \Delta_{m}\right) U_{a}-U_{1} \Delta_{m, a} \\
& 0 \\
& =\left(\begin{array}{c}
b_{m+1}^{(1)} v_{1} \\
0
\end{array}\right)-\left(\begin{array}{c}
-\alpha r h_{m} \\
\beta r h_{m}-\alpha r s h_{m}
\end{array}\right)+\left(\begin{array}{c}
(1) \\
-\alpha c_{m+1} v_{1}+\alpha r e_{m+1}+f_{m+1}
\end{array}\right) \\
& =\left(\begin{array}{c}
b_{m+1}^{(1)} v_{1}+\alpha r h_{m} \\
-\alpha c_{m+1}^{(1)} v_{1}+\alpha r e_{m+1}+f_{m+1}-\beta r h_{m}+\alpha r s h_{m}
\end{array}\right), m \geq 0 .
\end{aligned}
$$


Therefore, the $m$-th enlarged discrete zero curvature equation

$$
\bar{U}_{t_{m}}=\left(E \bar{V}^{[m]}\right) \bar{U}-\bar{U} \bar{V}^{[m]}
$$

leads to

$$
v_{t_{m}}=\left(\begin{array}{c}
v_{1} \\
v_{2}
\end{array}\right)_{t_{m}}=S_{m}(u, v)=\left(\begin{array}{c}
b_{m+1}^{(1)} v_{1}+\alpha r h_{m} \\
-\alpha c_{m+1}^{(1)} v_{1}+\alpha r e_{m+1}+f_{m+1}-\beta r h_{m}+\alpha r s h_{m}
\end{array}\right),
$$

together with the $m$-th generalized Toda equation in (3.8). Therefore, we obtain a hierarchy of coupling systems defined by (2.18):

$$
\bar{u}_{t_{m}}=\left(\begin{array}{c}
u \\
v
\end{array}\right)_{t_{m}}=\bar{K}_{m}(u)=\left(\begin{array}{c}
K_{m}(u) \\
S_{m}(u, v)
\end{array}\right), \quad m \geq 0
$$

for the generalized Toda hierarchy (3.8).

Let us second consider the semi-direct sum of Lie algebras of $4 \times 4$ matrices:

$G \in G_{c}, G=\left\{\left(\begin{array}{ll}A & 0 \\ 0 & A\end{array}\right) \mid A \in \mathbb{C}\left[\lambda, \lambda^{-1}\right] \otimes M_{2 \times 2}\right\}, G_{c}=\left\{\left(\begin{array}{ll}0 & B \\ 0 & 0\end{array}\right) \mid B \in \mathbb{C}\left[\lambda, \lambda^{-1}\right] \otimes M_{2 \times 2}\right\}$.

In this case, $G_{c}$ is an Abelian ideal of $G \in G_{c}$, too. We define the corresponding enlarged spatial spectral matrix as

$$
\bar{U}=\bar{U}(\bar{u}, \lambda)=\left(\begin{array}{cc}
U & U_{a} \\
0 & U
\end{array}\right) \in G \notin G_{c}, U_{a}=U_{a}(v)=\left(\begin{array}{cc}
v_{1} & v_{2} \\
v_{3} & v_{4}
\end{array}\right),
$$

where $v_{i}, 1 \leq i \leq 4$, are new dependent variables and

$$
v=\left(v_{1}, v_{2}, v_{3}, v_{4}\right)^{T}, \bar{u}=\left(u^{T}, v^{T}\right)^{T}=\left(r, s, v_{1}, v_{2}, v_{3}, v_{4}\right)^{T} .
$$

If we set

$$
\bar{W}=\left(\begin{array}{cc}
W & W_{a} \\
0 & W
\end{array}\right), W_{a}=W_{a}(\bar{u}, \lambda)=\left(\begin{array}{cc}
e & f \\
g & -e
\end{array}\right),
$$

where $W$ is a solution to $(E W) U-U W=0$, defined by (3.3), then the corresponding enlarged discrete stationary zero curvature equation $(E \bar{W}) \bar{U}-\bar{U} \bar{W}=0$ becomes

$$
(E W) U_{a}+\left(E W_{a}\right) U-U W_{a}-U_{a} W=0
$$

which is equivalent to

$$
\left\{\begin{array}{l}
\left(e^{(1)}+e\right)+(\lambda+s) f^{(1)}+\left(a^{(1)}+a\right) v_{2}+b^{(1)} v_{4}-b v_{1}=0, \\
-(\alpha \lambda+\beta) r\left(e^{(1)}+e\right)-(\lambda+s) g+(\alpha \lambda+\beta)\left(c^{(1)} v_{1}-c v_{4}\right)-\left(a^{(1)}+a\right) v_{3}=0, \\
g^{(1)}-(\lambda+s)\left(e^{(1)}-e\right)-(\alpha \lambda+\beta)\left(r f-c^{(1)} v_{2}\right)-\left(a^{(1)}-a\right) v_{4}-b v_{3}=0 .
\end{array}\right.
$$


This system can determine a solution for $e, f$ and $g$ as follows

$$
e=\sum_{i \geq 0} e_{i} \lambda^{-i}, f=\sum_{i \geq 0} f_{i} \lambda^{-i}, g=\sum_{i \geq 0} g_{i} \lambda^{-i}
$$

Now, we define the enlarged temporal spectral matrix as

$$
\bar{V}^{[m]}=\left(\begin{array}{cc}
V^{[m]} & V_{a}^{[m]} \\
0 & V^{[m]}
\end{array}\right), V_{a}^{[m]}=\left(\lambda^{m} W_{a}\right)_{+}+\Delta_{m, a}, m \geq 0,
$$

where $V^{[m]}$ is defined as in (3.6). To satisfy (2.17), choose $\Delta_{m, a}$ as

$$
\Delta_{m, a}=\left(\begin{array}{cc}
h_{m} & 0 \\
-\alpha r h_{m} & 0
\end{array}\right), h_{m}-\text { arbitrary, } m \geq 0 .
$$

Then based on (3.22), we can compute that

$$
\begin{aligned}
& \left(E V^{[m]}\right) U_{a}+\left(E V_{a}^{[m]}\right) U-U V_{a}^{[m]}-U_{a} V^{[m]} \\
& =\left[\left(E V_{m}\right) U_{a}+\left(E\left(\lambda^{m} W_{a}\right)_{+}\right) U-U\left(\lambda^{m} W_{a}\right)_{+}-U_{a} V_{m}\right] \\
& +\left[\left(E \Delta_{m}\right) U_{a}-U_{a} \Delta_{m}\right]+\left[\left(E \Delta_{m, a}\right) U_{1}-U_{1} \Delta_{m, a}\right] \\
& =\left(\begin{array}{cc}
-\alpha c_{m+1} v_{2}+\alpha r f_{m+1}^{(1)} & f_{m+1}^{(1)} \\
\alpha c_{m+1}^{(1)} v_{1}-\alpha c_{m+1} v_{4}-\alpha r\left(e_{m+1}^{(1)}+e_{m+1}\right)-g_{m+1} & \alpha c_{m+1}^{(1)} v_{2}-\alpha r f_{m+1}-\left(e_{m+1}^{(1)}-e_{m+1}\right.
\end{array}\right) \\
& +\left(\begin{array}{cc}
\left(b_{m+1}^{(1)}-b_{m+1}\right) v_{1} & b_{m+1}^{(1)} v_{2} \\
-b_{m+1} v_{3} & 0
\end{array}\right)+\left(\begin{array}{cc}
\alpha r h_{m} & h_{m}^{(1)} \\
r(\alpha s-\beta) h_{m} & -\alpha r^{(1)} h_{m}^{(1)}
\end{array}\right) \\
& =\left(\begin{array}{c}
-\alpha c_{m+1} v_{2}+\alpha r f_{m+1}^{(1)}+\left(b_{m+1}^{(1)}-b_{m+1}\right) v_{1}+\alpha r h_{m}, \\
\alpha c_{m+1}^{(1)} v_{1}-\alpha c_{m+1} v_{4}-\alpha r\left(e_{m+1}^{(1)}+e_{m+1}\right)-g_{m+1}-b_{m+1} v_{3}+r(\alpha s-\beta) h_{m},
\end{array}\right. \\
& f_{m+1}^{(1)}+b_{m+1}^{(1)} v_{2}+h_{m}^{(1)} \\
& \left.\alpha c_{m+1}^{(1)} v_{2}-\alpha r f_{m+1}-\left(e_{m+1}^{(1)}-e_{m+1}\right)-\alpha r^{(1)} h_{m}^{(1)}\right) \text {. }
\end{aligned}
$$

Then the $m$-th enlarged discrete zero curvature equation

$$
\bar{U}_{t_{m}}=\left(E \bar{V}^{[m]}\right) \bar{U}-\bar{U} \bar{V}^{[m]}
$$

leads to

$$
\begin{aligned}
& v_{t_{m}}=\left(v_{1}, v_{2}, v_{3}, v_{4}\right)_{t_{m}}^{T}=T_{m}(u, v) \\
& -\alpha c_{m+1} v_{2}+\alpha r f_{m+1}^{(1)}+\left(b_{m+1}^{(1)}-b_{m+1}\right) v_{1}+\alpha r h_{m} \\
& f_{m+1}^{(1)}+b_{m+1}^{(1)} v_{2}+h_{m}^{(1)} \\
& =\left(\begin{array}{c}
(1) \\
\alpha c_{m+1}^{(1)} v_{1}-\alpha c_{m+1} v_{4}-\alpha r\left(e_{m+1}^{(1)}+e_{m+1}\right)-g_{m+1}-b_{m+1} v_{3}+r(\alpha s-\beta) h_{m} \\
\alpha c_{m+1}^{(1)} v_{2}-\alpha r f_{m+1}-\left(e_{m+1}^{(1)}-e_{m+1}\right)-\alpha r^{(1)} h_{m}^{(1)}
\end{array}\right),
\end{aligned}
$$


together with the $m$-th generalized Toda equation in (3.8). Therefore, we obtain a hierarchy of coupling systems defined by (2.19):

$$
\bar{u}_{t_{m}}=\left(\begin{array}{c}
u \\
v
\end{array}\right)_{t_{m}}=\bar{K}_{m}(u)=\left(\begin{array}{c}
K_{m}(u) \\
T_{m}(u, v)
\end{array}\right), \quad m \geq 0
$$

for the generalized Toda hierarchy (3.8).

\subsection{Illustrative examples}

We now work out two concrete examples as follows, one in each of the two above cases.

Case of $\alpha=1$ and $\beta=0$ : Let us first compute an example of the hierarchy (3.19). Assume that $\alpha=1$ and $\beta=0$ for convenience, which corresponds to the lattice hierarchy presented in [26]. In this case, we have $n_{0}=0$ in (2.14).

It directly follows from (3.15) that

$$
\left\{\begin{array}{l}
f_{i}=a_{i}^{(1)} v_{1}+b_{i}^{(1)} v_{2} \\
r e_{i+1}=c_{i+1}^{(1)} v_{1}-a_{i}^{(1)} v_{2}-f_{i+1}-s f_{i}
\end{array}\right.
$$

where $i \geq 0$ and $r e_{0}=c_{0}^{(1)} v_{1}-f_{0}$. We can then obtain that

$$
\left\{\begin{array}{l}
f_{0}=-\frac{1}{2} v_{1}, f_{1}=r^{(1)} v_{1}+v_{2} \\
f_{2}=-\left[r^{(2)} r^{(1)}+\left(r^{(1)}\right)^{2}+r^{(1)} r+r^{(1)} s^{(1)}+r^{(1)} s\right] v_{1}-\left(r^{(1)}+r+s\right) v_{2} \\
r e_{0}=\frac{1}{2} v_{1}, r e_{1}=\frac{1}{2} s v_{1}-\frac{1}{2} v_{2}, r e_{2}=r^{(1)} r v_{1}+r v_{2} .
\end{array}\right.
$$

If we choose

$$
h_{1}=2 \xi f_{0} f_{1}=-\xi v_{1}\left(r^{(1)} v_{1}+v_{2}\right), \xi=\text { const. }
$$

then the vector-valued function $S_{1}$ defined by (3.18) becomes

$$
S_{1}(u, v)=\left(\begin{array}{c}
-\left(r^{(1)}+r+s\right) v_{1}-\xi r v_{1}\left(r^{(1)} v_{1}+v_{2}\right) \\
-r^{(1)} s v_{1}-\left(r^{(1)}+s\right) v_{2}-\xi r s v_{1}\left(r^{(1)} v_{1}+v_{2}\right)
\end{array}\right)
$$

Therefore, the integrable coupling of the generalized Toda lattice equation (3.11), defined by (3.19), reads as

$$
\left\{\begin{array}{l}
r_{t_{1}}=r\left(s^{(-1)}-s\right)+r\left(r^{(-1)}-r^{(1)}\right) \\
s_{t_{1}}=s\left(r-r^{(1)}\right) \\
v_{1, t_{1}}=-\left(r^{(1)}+r+s\right) v_{1}-\xi r v_{1}\left(r^{(1)} v_{1}+v_{2}\right) \\
v_{2, t_{1}}=-r^{(1)} s v_{1}-\left(r^{(1)}+s\right) v_{2}-\xi r s v_{1}\left(r^{(1)} v_{1}+v_{2}\right)
\end{array}\right.
$$


the second sub-system of which is nonlinear with respect to both sub-sets of dependent variables when $\xi \neq 0$.

Case of $\alpha=0$ and $\beta=1$ : Let us second compute an example of the hierarchy (3.26). Assume that $\alpha=0$ and $\beta=1$ for convenience, which corresponds to the Toda lattice hierarchy. In this case, we have $n_{0}=1$ in (2.14).

We take the initial set of functions as follows:

$$
f_{0}=g_{0}=0, e_{0}=-\frac{1}{2}
$$

Obviously from (3.22), we can have

$$
\left\{\begin{array}{l}
f_{i+1}^{(1)}=-s f_{i}^{(1)}-\left(e_{i}^{(1)}+e_{i}\right)-\left(a_{i}^{(1)}+a_{i}\right) v_{2}-b_{i}^{(1)} v_{4}+b_{i} v_{1}, \\
g_{i+1}=-s g_{i}-r\left(e_{i}^{(1)}+e_{i}\right)-\left(a_{i}^{(1)}+a_{i}\right) v_{3}+c_{i}^{(1)} v_{1}-c_{i} v_{4}, \\
e_{i+1}^{(1)}-e_{i+1}=-s\left(e_{i}^{(1)}-e_{i}\right)+g_{i}^{(1)}-r f_{i}-\left(a_{i}^{(1)}-a_{i}\right) v_{4}+c_{i}^{(1)} v_{2}-b_{i} v_{3},
\end{array}\right.
$$

where $i \geq 0$. It then follows that

$$
\left\{\begin{array}{l}
f_{1}=1+v_{2}^{(-1)}, g_{1}=r+v_{3}, e_{1}=v_{3}+r\left(1+v_{2}^{(-1)}\right) \\
f_{2}=-\left(s^{(-1)}+r\right)\left(1+v_{2}^{(-1)}\right)-v_{3}-v_{3}^{(-1)}-r^{(-1)}\left(1+v_{2}^{(-2)}\right)-v_{4}^{(-1)}+v_{1}^{(-1)} \\
g_{2}=-s\left(r+v_{3}\right)-r\left[v_{3}^{(1)}+v_{3}+r^{(1)}\left(1+v_{2}\right)+r\left(1+v_{2}^{(-1)}\right)\right]+r^{(1)} v_{1}-r v_{4} \\
e_{2}^{(1)}-e_{2}=(1-s)\left(r^{(1)}-r+v_{3}^{(1)}-v_{3}+r^{(1)} v_{2}-r v_{2}^{(-1)}\right) .
\end{array}\right.
$$

We can use the inverse formula (1.4) to compute $e_{2}$ here, but as we will see, this is not necessary for computing the corresponding integrable coupling.

Now if we choose

$$
h_{1}=\eta v_{1} e_{1}=\eta v_{1}\left[v_{3}+r\left(1+v_{2}^{(-1)}\right)\right], \eta=\text { const. }
$$

then the vector-valued function $T_{1}$ defined by (3.25) becomes

$$
T_{1}(u, v)=\left(\begin{array}{c}
-\left(s-s^{(-1)}\right) v_{1} \\
-s v_{2}-\left(s+r^{(1)}\right)\left(1+v_{2}\right)-r\left(1+v_{2}^{(-1)}\right)+v_{1}-v_{3}^{(1)} \\
-v_{3}-v_{4}+\eta v_{1}^{(1)}\left[v_{3}^{(1)}+r^{(1)}\left(1+v_{2}\right)\right] \\
\left(s^{(-1)}+s\right) v_{3}+r s+r\left[v_{3}^{(1)}+v_{3}+r^{(1)}\left(1+v_{2}\right)+r\left(1+v_{2}^{(-1)}\right)\right] \\
-r^{(1)} v_{1}+r v_{4}-\eta r v_{1}\left[v_{3}+r\left(1+v_{2}^{(-1)}\right)\right] \\
(s-1)\left(r^{(1)}-r+v_{3}^{(1)}-v_{3}+r^{(1)} v_{2}-r v_{2}^{(-1)}\right)
\end{array}\right)
$$


Therefore, the integrable coupling of the Toda lattice equation (3.10), defined by (3.26), reads as

$$
\left\{\begin{aligned}
r_{t_{1}}= & r\left(s^{(-1)}-s\right), s_{t_{1}}=r^{(1)}-r \\
v_{1, t_{1}}= & -\left(s-s^{(-1)}\right) v_{1}, \\
v_{2, t_{1}}= & -s v_{2}-\left(s+r^{(1)}\right)\left(1+v_{2}\right)-r\left(1+v_{2}^{(-1)}\right)+v_{1}-v_{3}^{(1)} \\
& -v_{3}-v_{4}+\eta v_{1}^{(1)}\left[v_{3}^{(1)}+r^{(1)}\left(1+v_{2}\right)\right], \\
v_{3, t_{1}}= & \left(s^{(-1)}+s\right) v_{3}+r s+r\left[v_{3}^{(1)}+v_{3}+r^{(1)}\left(1+v_{2}\right)+r\left(1+v_{2}^{(-1)}\right)\right] \\
& -r^{(1)} v_{1}+r v_{4}-\eta r v_{1}\left[v_{3}+r\left(1+v_{2}^{(-1)}\right)\right], \\
v_{4, t_{1}}= & (s-1)\left(r^{(1)}-r+v_{3}^{(1)}-v_{3}+r^{(1)} v_{2}-r v_{2}^{(-1)}\right),
\end{aligned}\right.
$$

the second sub-system of which is nonlinear with respect to both sub-sets of dependent variables when $\eta \neq 0$.

\section{Conclusions and remarks}

A feasible approach to construct integrable couplings of discrete soliton equations has been proposed by taking advantage of semi-direct sums of Lie algebras, and the resulting theory has been applied to the generalized Toda hierarchy of lattice equations to generate integrable couplings for the hierarchy. The key point in our generating scheme is to establish a relation between semi-direct sums of Lie algebras and integrable couplings of discrete soliton equations. The underlying discrete matrix spectral problems are generated from semi-direct sums of Lie algebras, and the discrete Lax spectral matrices associated with given soliton equations play the non-ideal part in the semi-direct sums.

In our analysis of the two specific semi-direct sums, we have seen that there is always an arbitrary modified term $\Delta_{n, a}$. This indicates that higher order matrix spectral problems have more degrees of freedom in generating integrable systems. On the other hand, in all additional spectral sub-matrices such as $U_{a_{i}}$, one can take their dependence on the spectral parameter into consideration, and this will bring much more diverse integrable couplings.

There are also other questions about integrable properties of the resulting enlarged lattice equations, even in the case where additional spectral sub-matrices are independent of the spectral parameter. For example, can we solve the enlarged lattice equations by the inverse scattering transform? The class of Lie algebras in (2.8) provides a few realizations of semi-direct sums of Lie algebras. Other possible realizations are still interesting, especially those which could carry significant information about integrable properties. Reductions of the presented cases of semi-direct sums, which keep the uniqueness property of discrete spectral problems (see [17]), could be good examples. 
We would especially like to emphasize that we have been considering the problem of integrable couplings and the key is semi-direct sums of Lie algebras. The initial Lie algebras $G$ associated with given integrable systems in our construction can be simple (e.g., see [29, 30]), but semi-direct sums of Lie algebras $\bar{G}$ are normally non-simple (see [15]). Our examples in Section 2 are all non-simple, since the Killing forms on those semi-direct sums of Lie algebras $\bar{G}$ are degenerate. However, there still exist specific non-degenerate bilinear forms on the Lie algebras $\bar{G}$, with nice invariance properties, and their corresponding generalized trace identities, which present Hamiltonian structures of the enlarged lattice equations.

To conclude, semi-direct sums of Lie algebras provide a good source of matrix spectral problems for generating integrable systems, and thus the study of integrable couplings using semi-direct sums of Lie algebras will enhance our understanding of classification of integrable systems. We are expecting to see more research on related topics.

Acknowledgements: This work was supported in part by the University of South Florida Internal Awards Program under Grant No. 1249-936RO and by the National Science Foundation of China (Grant no. 10471139). Financial support from Dean's office of the College of Arts and Sciences of the University of South Florida is also gratefully acknowledged.

\section{References}

[1] W. X. Ma and B. Fuchssteiner, "Integrable theory of the perturbation equations," Chaos, Solitons \& Fractals 7, 1227-1250 (1996).

[2] W. X. Ma, "Integrable couplings of soliton equations by perturbations I - A general theory and application to the KdV hierarchy," Methods Appl. Anal. 7, 21-55 (2000).

[3] W. X. Ma and B. Fuchssteiner, "The bi-Hamiltonian structure of the perturbation equations of the KdV hierarchy," Phys. Lett. A 213, 49-55 (1996).

[4] W. X. Ma, "Enlarging spectral problems to construct integrable couplings of soliton equations," Phys. Lett. A 316, 72-76 (2003).

[5] W. X. Ma, "Integrable couplings of vector AKNS soliton equations," J. Math. Phys. 46, 033507, 19pp (2005).

[6] F. G. Guo and Y. Zhang, "A new loop algebra and a corresponding integrable hierarchy, as well as its integrable coupling," J. Math. Phys. 44, 5793-5803 (2003).

[7] Y. Zhang, "A generalized multi-component Glachette-Johnson (GJ) hierarchy and its integrable coupling system," Chaos, Solitons \& Fractals 21, 305-310 (2004).

[8] L. Frappat, A. Sciarrino and P. Sorba, Dictionary on Lie Algebras and Superalgebras (Academic Press, Inc., San Diego, CA, 2000). 
[9] S. Yu. Sakovich, "On integrability of a $(2+1)$-dimensional perturbed KdV equation," $J$. Nonlinear Math. Phys. 5, 230-233 (1998).

[10] S. Yu. Sakovich, "Coupled KdV equations of Hirota-Satsuma type," J. Nonlinear Math. Phys. 6, 255-262 (1999).

[11] W. X. Ma and R. G. Zhou, "Nonlinearization of spectral problems for the perturbation KdV systems," Physica A 296, 60-74 (2001).

[12] W. X. Ma and R. G. Zhou, "Binary nonlinearization of spectral problems of the perturbation AKNS systems," Chaos, Solitons \& Fractals 13, 1451-1463 (2002).

[13] W. X. Ma, "A bi-Hamiltonian formulation for triangular systems by perturbations," J. Math. Phys. 43, 1408-1421 (2002).

[14] E. G. Fan and Y. Zhang, "A simple method for generating integrable hierarchies with multipotential functions," Chaos, Solitons \& Fractals 25, 425-439 (2005).

[15] W. X. Ma, X. X. Xu and Y. Zhang, "Semi-direct sums of Lie algebras and continuous integrable couplings," Phys. Lett. A 351, 125-130 (2006).

[16] G. Z. Tu, "A trace identity and its applications to the theory of discrete integrable systems," J. Phys. A: Math. Gen. 23, 3903-3922 (1990).

[17] W. X. Ma and B. Fuchssteiner, "Algebraic structure of discrete zero curvature equations and master symmetries of discrete evolution equations," J. Math. Phys. 40, 2400-2418 (1999).

[18] M. J. Ablowitz and J. F. Ladik, "Nonlinear differential-difference equations," J. Math. Phys. 16, 598-603 (1975).

[19] W. X. Ma and X. X. Xu, "A modified Toda spectral problem and its hierarchy of biHamiltonian lattice equations," J. Phys. A: Math. Gen. 37, 1323-1336 (2004).

[20] H. W. Tam and Y. Zhang, "A new algebraic system and its applications," Chaos, Solitons \& Fractals 23, 151-157 (2005).

[21] H. X. Yang, X. X. Xu and H. Y. Ding, "New hierarchies of integrable positive and negative lattice models and Darboux transformation," Chaos, Solitons \& Fractals 26 1091-1103 (2005).

[22] M. Toda, Theory of Nonlinear Lattices 2nd edition, Springer Series in Solid-State Sciences 20 (Springer, Berlin, 1989).

[23] T. Tsuchida, H. Ujino and M. Wadati, "Integrable semi-discretization of the coupled modified KdV equations," J. Math. Phys. 39, 4785-4813 (1998).

[24] T. Tsuchida, H. Ujino and M. Wadati, "Integrable semi-discretization of the coupled nonlinear Schrödinger equations," J. Phys. A: Math. Gen. 32, 2239-2262 (1999).

[25] D. J. Zhang and D. Y. Chen, "The conservation laws of some discrete soliton systems," Chaos, Solitons \& Fractals 14, 573-579 (2002).

[26] W. X. Ma and X. X. Xu, "Positive and negative hierarchies of integrable lattice models associated with a Hamiltonian pair," Internat. J. Theoret. Phys. 43, 219-235 (2004). 
[27] S. V. Manakov, "Complete integrability and stochastization of discrete dynamical systems," Soviet Physics JETP 40, 269-274 (1975).

[28] H. Flaschka, "The Toda lattice I - Existence of integrals," Phys. Rev. B 9, 1924-1925 (1974).

[29] O. I. Bogoyavlensky, "On perturbations of the periodic Toda lattice," Comm. Math. Phys. 51, 201-209 (1976).

[30] M. A. Olshanetsky and A. M. Perelomov, "Classical integrable finite-dimensional systems related to Lie algebras," Phys. Rep. 71, 313-400 (1981).

[31] W. X. Ma and Y. You, "Rational solutions of the Toda lattice equation in Casoratian form," Chaos, Solitons \& Fractals 22, 395-406 (2004).

[32] W. X. Ma and K. Maruno, "Complexiton solutions of the Toda lattice equation," Physica A 343, 219-237 (2004). 